Rainer E. Zimmermann:

\title{
An Integral Perspective of Social Action: Imagining, Assessing, Choosing (Onto-epistemology of Networks)
}

\begin{abstract}
:
Starting from a formal and abstract perspective, the concept of networks is introduced with a view to possible connections to other fields of the sciences and to practical applications. The structural hierarchy of forms is identified expressing the conceptual organization of our observable world. In the case of social networks, it can be shown that they exhibit a characteristic type of self-reference, a result of their special relationship to the conditions of the human modes of cognition and communication. As to a possible derivation of strategic attitudes, it can be shown that a re-vitalization of the ancient concept of kalokagathía could turn out to be helpful in tackling present everyday problems. Hence, choosing the perspective of an explicit network paradigm entails a new reconciliation of aesthetics and ethics, respectively, including multifarious implications for a suitable foundation of praxis within pertinent crisis management.
\end{abstract}

Dedicated to the memory of Jon Lord.

\section{Agenda:}

Introduction

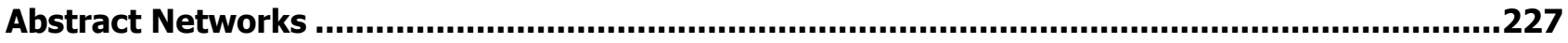

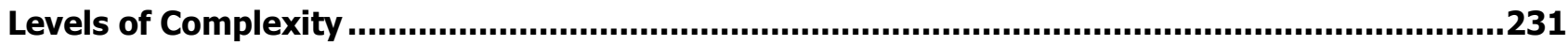

Social Networks $\ldots \ldots \ldots \ldots \ldots \ldots \ldots \ldots$

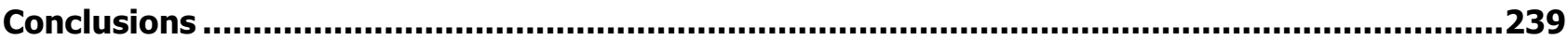

Author:

Prof. Dr. Dr. (habil.) Rainer E. Zimmermann

- Hochschule München (University of Applied Science), Dachauerstr. 100A, 80663 München, Germany

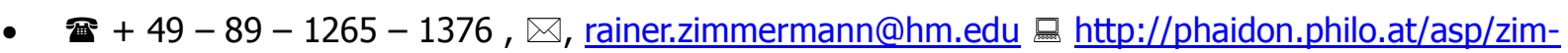
mermann.htm

- Relevant publications:

- System des transzendentalen Materialismus, Mentis, Paderborn, 2004

- New Ethics Proved in Geometrical Order. Spinozist Reflexions on Evolutionary Systems. Emergent Publications, Lichtfield Park, AZ, USA, 2011 


\section{Introduction}

The concept of network shows up as one of the universal concepts common both in the scientific discourse as well as in the colloquial language of everyday's discourse, respectively. ${ }^{1}$ In the latter case, as is usually true for most of the colloquial concepts utilized in a somewhat non-systematic manner, cause and effect are often mixed up: Indeed, as it turns out, and as we will show in this lecture course in some more detail, the concept of network is an instrument of representational techniques in the first place - rather than something that would be existing independently within the world. In other words: Networks are means of representation and mapping, and they are thus modes of human cognition that employs them for modelling purposes, but they are not properties of nature itself, i.e. structures that would also exist, if nobody would be around capable of observing them. While the scientific discourse is dealing with the conceptualization of phenomena on all levels of complexity, the discourse of everyday life is dealing with social aspects of the world (i.e. with the highest known level of complexity) only, though in a somewhat non-epistemic and at most fuzzy and approximate manner. This is the reason for the former being explicitly abstract as compared with the latter. Note that therefore, the word concrete utilized as the opposite of the word abstract signifies nothing but the cognitive conditions of human modality as visualized in common (i.e. non-scientific) terms.

In order to illuminate this relationship in more detail, we will start here (in section 2) with introducing the concept of network in a purely abstract manner and in formal terms. We will see that this turns out to be helpful for stressing the universality of the concept. It will also help to clarify the interrelationship among other concepts such as those of space (and time as to that) and system. We will discuss then (in section 3) the universality of the concepts themselves by displaying the various, hierarchically ordered levels of complexity within observable nature. When we arrive (in section 4) at the highest level (of social systems), it is time to note the explicit selfreference of the concepts discussed so far. This will help to solve most of the questions raised above when mentioning the mixing up of cause and effect as to the concept of network.

This discussion deals mainly with an onto-epistemological view (in the sense of Sandkühler) to the questions raised here. In a sense, it is the anthropological perspective onto which the conceptualization of scientific methods is actually grounded. Consequently, our conclusions will unfold a field of possibilities with a view to adequate human reflexion and action which might turn out to be useful in order to eventually develop strategic procedures that prove relevant for a succeeding daily life by bringing together the latter's aesthetical as well as ethical aspects. We thus find that the ancient philosophical concept of kalokagathía gains a renewed significance after all.

\section{Abstract Networks}

Essentially, a network is simply a set of nodes and links such that there is a characteristic structure of connections among the nodes. This can be easily drawn in the following manner (thanks to wikipedia indeed! ${ }^{2}$ ):

\footnotetext{
1 For a general overview see in detail Rainer E. Zimmermann: System des transzendentalen Materialismus, Mentis, Paderborn, 2004. My first paper on the topic is possibly id.: Netzwerkstrukturen und die Emergenz des Neuen. In: Johannes Ehrhardt (ed.), Netzwerk-Dimensionen, Datacom, Bergheim, 1992, 70-85.
}

2 http://en.wikipedia.org/wiki/Graph_theory (2012-07-12) 


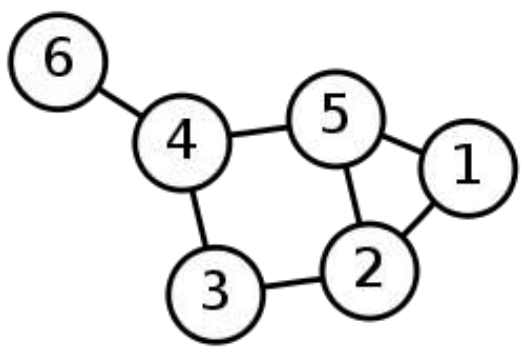

Figure 1: Example of representation of a simple network

Obviously, nodes 1 through 6 are connected here according to a number of given links. Note that what we see in the drawing is in fact, a representation of the network rather than the network itself. Hence, what we deal with is actually a mathematical graph, and as such we define this as a set of vertices and edges. Instead of doubling the work by doing so, this procedure of differing between the concrete object and its representation has the advantage of simplifying the usually complex phenomenology in order to derive sufficiently general results about properties of these configurations. So what the abstraction that goes hand in hand with representation actually achieves is a kind of universalization. We see this clearly by observing that we have not at all defined the meaning of nodes and links so far. Up to now, we simply deal with a formaltype of representation.

Connection in nature usually implies interaction. This is the reason for visualizing links in a network as expression for interactions among nodes. The idea is that the dynamics of an observable phenomenology is inherent in a diagram which is static itself. Thanks to the advent of computer graphics we are nowadays in a position to actually model dynamical phenomena by simulating them as a kind of movie. Nevertheless, in principle, the main point of the diagrammatic graphism is to express what cannot be expressed by a drawing alone: motion. It is the link in a network, or the edge of its representation, that stands for this motion. One of the consequences is that we usually deal with diagrams whose vertices are just points and whose edges are directed lines (commonly indicated by the head of an arrow). This is because the vertices are visualized as agents that operate onto other agents by means of their respective interaction. So what we can formally do is to characterize the type of agent and the type of interaction. This is usually done in terms of numbers that codify (label or colour) a given quality of types. But we have to differ between internal and externaltypes of interaction. This can be seen utilizing a famous example from mathematics: Graph theory was practically invented by Leonard Euler who in 1736 published a paper on the bridges of the city of Königsberg as displayed in the following (oncemore thanks to wikipedia! ${ }^{3}$ :

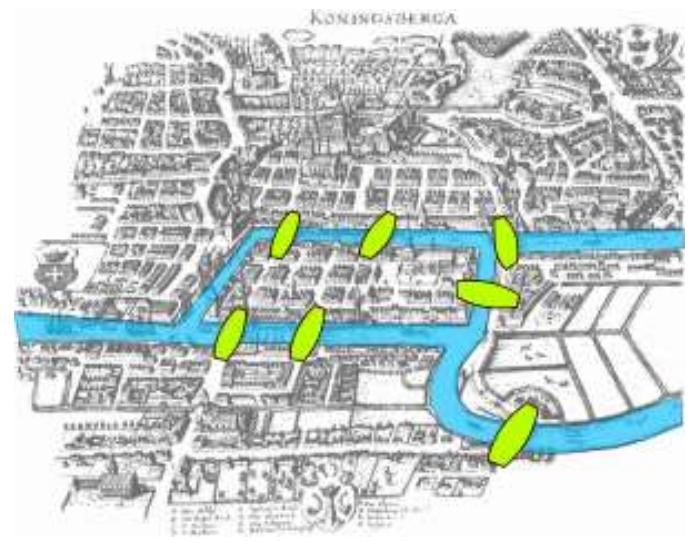

Figure 2: Representation of Euler's problem on the bridges of Königsberg

3 Ibid. 
Euler's idea was to ask whether it is possible to find a round trip through the city such that a person would have to cross each of the seven bridges only once. What Euler did was to abstract the city by shrinking the locations to points and expressing the bridges as links between points. So what we have then is a network with four nodes and seven links represented by a graph with four vertices and seven edges. Euler showed that nodes with an odd number of links must be either starting or end points of the journey. A continuous path that goes through all the bridges can have only one starting and one end point. Hence, such a path cannot exist on a graph that has more than two vertices with an odd number of edges. As the Königsberg graph has four such vertices, there cannot be a path of the desired kind. (Indeed, the people of Königsberg built a new bridge in 1875 that increased the number of edges of two of the vertices (in the sketch above the top and the bottom part of locations) to four. And that actually solved the problem.)

We learn two things here: First of all, we find that graph theory is actually dealing with topology. This is because the problem discussed above deals with connections of spatial parts. Indeed, if we visualize the whole city as an urban space, then the connections of this space constitute its topology. This is also why Euler thus inspired the analysis situs of Leibniz, forerunner of modern topology. And in fact, topological problems of this sort are not mere theoretical problems, but have explicit practical applications, for instance in the field of transport of goods and/or persons under an economic perspective.

But second, we recognize here the difference of internal and external interactions: The city map is a graphic layout that describes the interaction among nodes only in so far as this interaction can be potentially utilized, if necessary. That is, the bridges of Königsberg (visualized within a given network of streets) can be used by persons to their own purposes. But without persons, they represent nothing but a potential that can be eventually actualized. In other words: The nodes (vertices) do not represent active agents, and the operation of one agent onto the other is not active, but entailed by the topology of the space and thus passive only. This is why we differentiate interactions here as internal (if caused by active agents) and external (if caused by different active agents that utilize passive agents). Obviously, this difference also implies a difference of representations: namely whether they are utilized in order to map potentialities (as in the case of geographic maps e.g.) or actualities (as in the case of dynamic processes, e.g. concerning the communication between persons). It is straightforward to realize that in any case the diagrams serve the purpose of conceptualizing potential or actual processes.

A network of active interaction can be expressed for instance, in terms of a communication network that defines a small world model common for a large class of phenomena in the fields of communication, epidemics, information exchange and so on. (One has always to sort out where the active agent is operating and what is the passive layout as space of interactions.) The following diagram is displaying a small world that is established by acquaintances of persons. The idea is to display first-order interactions (persons who know persons personally) and mediated higher-order interactions (persons who know persons who know persons). Obviously, because the number of people on this planet is smaller than the range of possible higher-order interactions, one can show that all people are connected to each other via a line of six other persons on this chain of acquaintances. In other words, the world is small in the sense that interactions of this type cover the whole set of people on Earth up to sixth-order contact. (In fact, the secret lies partially in the possible shortcuts that are encountered when two persons meet who live in different continents, because the acquaintances of one person are to the other of second order without making it necessary for that person to actually ever visit the continent of the other person.) 


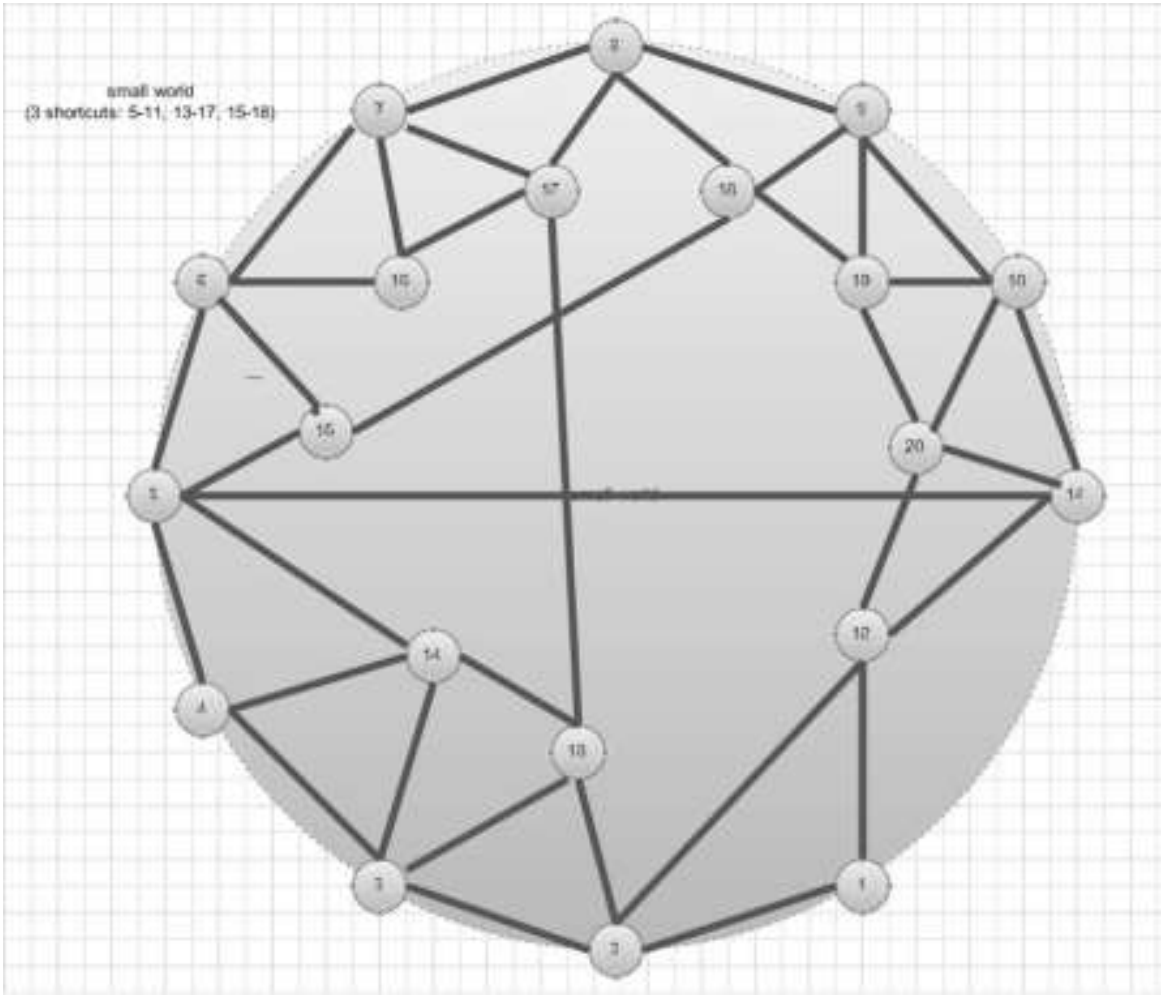

Figure 3: "Small World" Network"

We can easily see that similar networks are quite abundant: They can describe co-authorship of scientific articles (Erdös-Rényi), networks of lending money, the spreading of infectious deseases (percolation) and so on. Within this variety of networks, it is important to differ between those which are randomly scaled and those which are scale-free: One can show that if we start with $\mathrm{N}$ nodes and connect every pair of nodes with probability $\mathrm{p}$, then we create a graph with approximately $\mathrm{pN}(\mathrm{N}-1) / 2$ edges distributed randomly. ${ }^{5}$ The generated network is strongly homogeneous then (most nodes have approximately the same number of links). If we consider the degree distribution of a network $\mathrm{P}(\mathrm{k})$ : which is the probability that a randomly selected node has exactly $\mathrm{k}$ links - then we find that the degrees in a random graph follow a Poisson distribution with a peak at $<k>$. However, for a very large class of networks, we find instead that $P(k) \sim k^{-\gamma}$, i.e. the degree distribution possesses what we call a power-law tail. (Mostly then, $0<\gamma<1$, and the associated phase space dimension is thus fractal.) Such networks are strongly inhomogeneous: most nodes have one or two links, while a few nodes (called hubs) have a larger number of links that guarantee the network's overall connectivity. Hence, there is a major topological difference between random and scale-free networks. Examples of the latter include the www, metabolic and protein networks, language as well as networks of sexual reproduction.

Before looking more closely to the variety of networks two further remarks are in order as to representational techniques: The first concerns the fact that in mathematical terms, directed graphs (and thus linked networks) can be visualized as categories whose objects are the vertices (nodes) and whose morphisms are the edges (links). Note that as compared with sets, categories can be thought of as a dynamical generalization of sets, because while the latter are static and consist always of the same elements by which they are defined, the former express motion (evolution or change) of objects by means of their morphisms. While sets are mapped onto each other by mapping the elements of one set to elements of the other, categories are mapped onto

4 Illustration taken from: Bordalier Institute (Peter Winiwarter): www.bordalierinstitute.com

5 Here and elsewhere we follow the ground-breaking Stefan Bornholdt, Heinz Georg Schuster (eds.): Handbook of Graphs and Networks, Wiley-VCH, Weinheim, 2003. For a more general viewpoint see Albert-László Barabasi: Linked. The New Science of Networks. Perseus, Cambridge (Mass.), 2002. 
each other by mapping objects to objects and morphisms to morphisms. Mappings of sets are called functions. Mappings of categories are called functors. Usually, the set of functors is itself a category. Categories must fulfil the requirements of a different set of axioms as compared with sets, especially as to identity mappings and the composition of mappings. The complete formal apparatus of categories can thus be applied to networks which turns out to be quite helpful, for instance in the case of interpreting the network in terms of its adjacency matrix which expresses the characteristic of the relevant links such as their degree, or in the case of determining shortest paths within the network or hubs, relevant properties that define the robustness of networks.

The second point concerns the intrinsic isomorphism of networks and cellular automata: The latter is a computer programme that unfolds given cellular structures according to a set of simple rules. The updating can be accelerated so as to perform a movie of its evolution. The most famous (and at the same time the most simple) automaton of this kind is "Conway's game of life" that can achieve considerable complexity when starting with simple initial forms and following three equally simple rules that define the state of a cell with respect to the states of the neighboring cells. The cells can be quadratic or hexagonal or whatsoever. Usually, the geometric shape of the cell relates to the action undertaken, because mathematically, this can be described by a significant group action whose matrix representation (i.e. a collection of characteristic numbers) can be utilized in order to label (or colour) the underlying graph of the network. If one shrinks a cell to its central point and replaces the neighboring boundaries by links, the isomorphism to networks is quite apparent. In a sense, cellular automata can be visualized as practical applications of networks and their graphs.

\section{Levels of Complexity}

Now, the available time is too short ${ }^{6}$ so that we cannot dwell in more detail on the various phenomena that can be modelled in terms of networks. But it should have become apparent that all these phenomena can be classified according to their degree of complexity. In a sense, complexity turns out to be a quantitative measure of networks, because it essentially relates the number of possible connections in a network to the number of actualized connections. This number defines the actual state of the network. This more intuitive and combinatorial measure of complexity can be easily related to other more formal measures such as Kolmogorov's definition of complexity. Note however the following: If the quotient in question is $c=a / p$ (actualized over possible connections between nodes), then it can be normed such that the result is between 0 and 1 , respectively, in a straightforward manner: If all possible connections are actualized, then obviously, $c=1$. If no possible connection is actualized, then $c=0$. The usual value will be somewhere in between. The rest of non-actualized (but possible) connections is respectively, $r=(p-a) / p$ such that $c+r=1$. We call $r$ the network's redundancy. The latter is not quite a superflous or useless property, but much to the contrary, is important for the network's stability, because it defines a kind of space of free play within which the network has still some freedom to develop further.

What we have then, is essentially a hierarchy of structures in several big steps that originates from large state transitions of networks, when the quantity of connections spontaneously turns into new qualities: We have thus the physical level, the chemical level, the biological level, and so forth. All these levels are sub-structured somehow: Life for instance, can be visualized with a view to the molecular level, the cellular level, the organic level, and the population level, respectively. But the important point is here that all what there is consists of "the same stuff" (essentially matter, that is what can be visualized as that sort of energy that has acquired mass). In fact, the specific situation of physical energy is always accompanied by an equally specific situation of information. Information that is actualized in material forms is called structure then. Hence, the respective levels of the mentioned hierarchy are determined by a quantitative measure of complexity that is expressed in terms of characteristic qualitative properties of forms.

Within this hierarchy, the most fundamental level of discussion is the level of the physics of quantum gravity on which the basic categories of physics, i.e. space and time, as well as matter, can be represented by quantized

6 Note that this paper is the written version of a talk delivered to a summer school at the university of León in September 2012. 
entities. This is done in terms of the spin network, a purely combinatorial structure originally introduced by Roger Penrose (fig. 4).

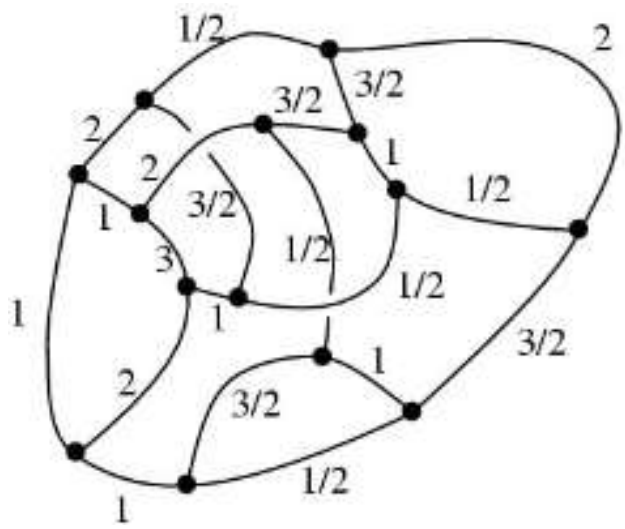

Figure 4: Spin Network ${ }^{7}$

The nodes of this network are active agents here, operators in fact that act upon their neighbours by exchanging spin numbers (normalized quantities of spin angular momentum which are expressed here in units of Planck's constant h). Hence, these agents can be visualized as abstract particles that operate by means of a computational algorithm according to given combinatorial rules. The decisive insight of Penrose is that a progressive condensation of portions of this spin network can be understood as the onset of physical classicity such that the dimensions of Euklidean space emerge spontaneously by a superposition of many layers of this network. Hence, if arranged in a hexagonal network accompanied by an equivalent triangulation of the underlying space of representation, the segments of this "field of spatial possibilities" represent the smallest portion of space in terms of its area and volume. As it turns out, this area is proportional to the square of the Planck length, while the volume is proportional to the third power of the Planck length. This is given by

$$
\ell_{\mathrm{P}}=\sqrt{\frac{\hbar G}{c^{3}}} \approx 1.616199(97) \times 10^{-35} \mathrm{~m}
$$

and defines the limit of physics in representing the smallest possible length scale at which a theory of everything is expected to be valid, presently conjectured as a future theory of quantum gravity. What we call "space" (and time as to that) is nothing but a multiple (superposition) of quantized portions of this fundamental type.

In fact, Lee Smolin has shown earlier that each hexagon in the spin network can be visualized as the cooperative result of six interacting loops that merge in the process. A loop is essentially the most fundamental agent in physics consisting of a parallel transportation device for a gauge field in microscopic terms, and it can be shown that this agent satisfies Stuart Kauffman's condition that it be able to perform at least one thermodynamic work cycle. (This is why this field is actually called loop quantum gravity.) As we would expect from what has been said before, after merging, one loop is represented by one vertex in the graph. Six vertices constitute one hexagon in the spin network. Hence, loops are agents that exist primordially before any space fragments are being formed. They are thus truly pre-geometric. (Look at the following for illustration.) 


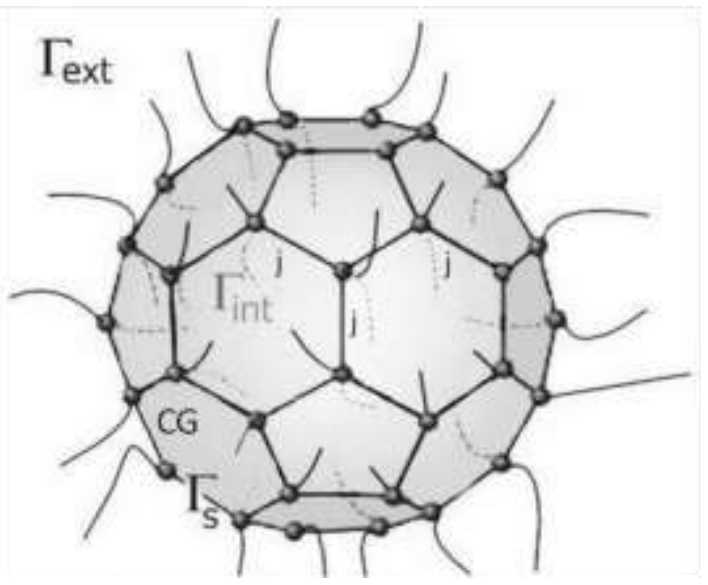

Figure 5: A quantized black hole

In other words, the physicist shows up as a somewhat "short-sighted" observer not sensitive for the detailed quantum structure of the world, but only for its classical average phenomenology. The physical universe can thus be visualized as a quantum computer that emerges in the cooperation of loops in groups of six and that computes (i.e. organizes and interprets) spin numbers all the time which is the most fundamental type of information about the physical world. But human beings who are part of the same universe (and are to the universe therefore what a sub-routine is to a master programme) cannot actually cognitively perceive the universe as it is, but only observe it according to their mode of being which is determined by the cognitive capacity that is developed within their own biology - itself the outcome of the underlying physics after all.

Note that the aspect of computation can be made quite explicit, because, as it turns out, the spin numbers encountered in the spin network above can at the same time be identified with the matrix representation of the group $S L(2, C)$ which is the special linear group of $2 \times 2$ matrices with complex entries (responsible for what we call spinors in Dirac's quantum physics). As John Baez has pointed out some time ago, the fact that this group has complex entries consisting of real and imaginary parts leads to the conclusion that the information processing underlying the exchange of spin numbers in the network can be visualized as quantum rather than classical computation, because it is not cbits that are processed here, but quantum bits (qubits). Hence, the idea that the universe can be visualized as a quantum computer. The same is more or less true for the other levels of the hierarchy: Thus chemical networks show up as superpositions of physical networks, biological networks as superpositions of chemical networks, and so on. Look first at a comparatively simple cycle which is transforming hydrogen into helium within the interior core of massive stars ${ }^{9}$ :

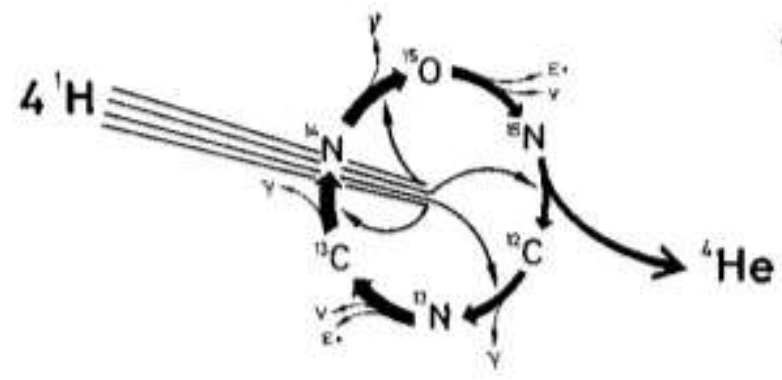

Figure 6: Carbon cycle, responsible -at least in part- for the energy production of massive stars, as proposed by Bethe and Weizsäcker ${ }^{12} \mathrm{C},{ }^{13} \mathrm{~N},{ }^{13} \mathrm{C},{ }^{14} \mathrm{~N},{ }^{15} \mathrm{O}$, and ${ }^{15} \mathrm{~N}$ are steadily reconstituted by cyclic reaction. The cyclic scheme as a whole represents a catalyst which converts four ${ }^{1} \mathrm{H}$ atoms to one ${ }^{4} \mathrm{He}$ atom, with the release of energy in form of $\gamma$-quanta, positrons $\left(\varepsilon^{+}\right)$and neutrinos $(v) .{ }^{10}$

8 The illustration is taken from M.H. Ansari: Gauge Invariance: www.gaugeinvariance.blogspot.com.

9 We utilize here and in the following again material of the Bordalier institute.

10 Illustration taken from the Webpage of Bordalier Institute, www.bordalierinstitute.com. 
The cyclic organization of directed interactions are quite common for natural processes. Probably, they are the pre-condition for the possibility of creating higher-order structures from lower-order structures. Hence, cycles belong to the foundation of the evolving increase of complexity. Things become even more complex: figure 7 shows the yeast protein interaction network. On the one hand, this is most interesting because of its scale-free structure that is common for self-organized criticality. On the other hand, it is this structure which is universal for all living organisms. (Note the clear display of hubs in the network's structure.)

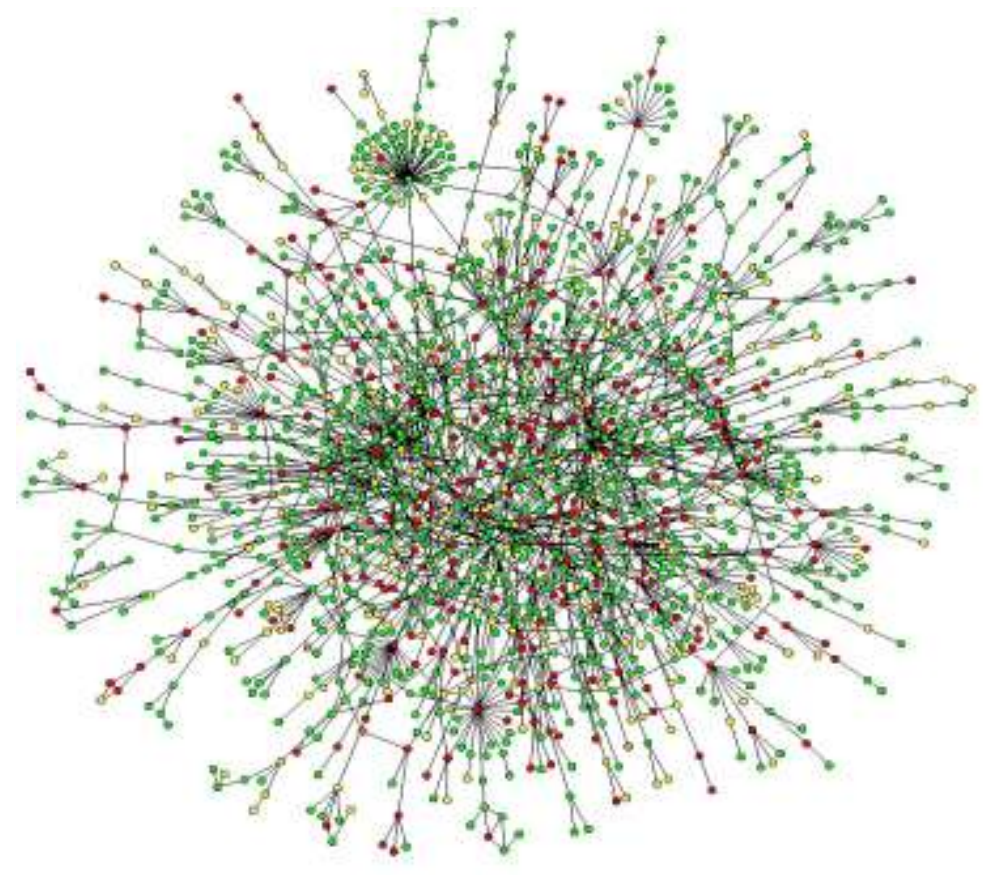

Figure 7: The yeast protein interaction network ${ }^{11}$

From the micro-structure of living beings to their macro-structure within the evolution on this very planet: Note in figure 8 the large space of free play as to possible variations of forms. Nevertheless, the network is essentially unbroken and well-connected. On the large scale structure of the planetary biosphere, very many species show up, but the outcome appears to be more or less homogeneous. It may be that humans define an explicit "hub" within the network, but if this is the case, then they do so on the social level which is beyond the scope of this diagram. Note however, that initially, "sociality" (or, language rather) is a new biologicalselective feature, and in the beginning, it is far from clear whether it will become dominant or not in the long run.

The point is that the organizational structure turns out to be invariant: We have agents (operators) that act upon their neighbours within networks connected by links that can be represented in terms of mathematical graphs. The phenomena can be classified then according to the internal and external qualities of interactions equivalent with links. Obviously, on the more fundamental levels (for instance within physics), the agents are genuinely active, because they do not only interact with their neighbours, but by doing so, also co-create their mutual environment, i.e. they define the layout on which they actually unfold in the first place. Speaking of agents, one can easily utilize the terminology of game theory in order to cover the interactions involved: There are the players, the space of free play ( = arena), the rules. In evolutionary terms, there are also winners and losers. Under a philosophical perspective, we can visualize these fundamental agents acting like players in a game as a kind of primordial subjectivity. In a sense, we can speak of proto-consciousness here such that these fundamental interactions are in fact actions according to decision rules, though not in a human sense, but on a very elementary level instead. Hence, on this lowest level of organization, the available agents have nevertheless their choice. And thus there is (proto-) meaning already.

11 Ibid. 


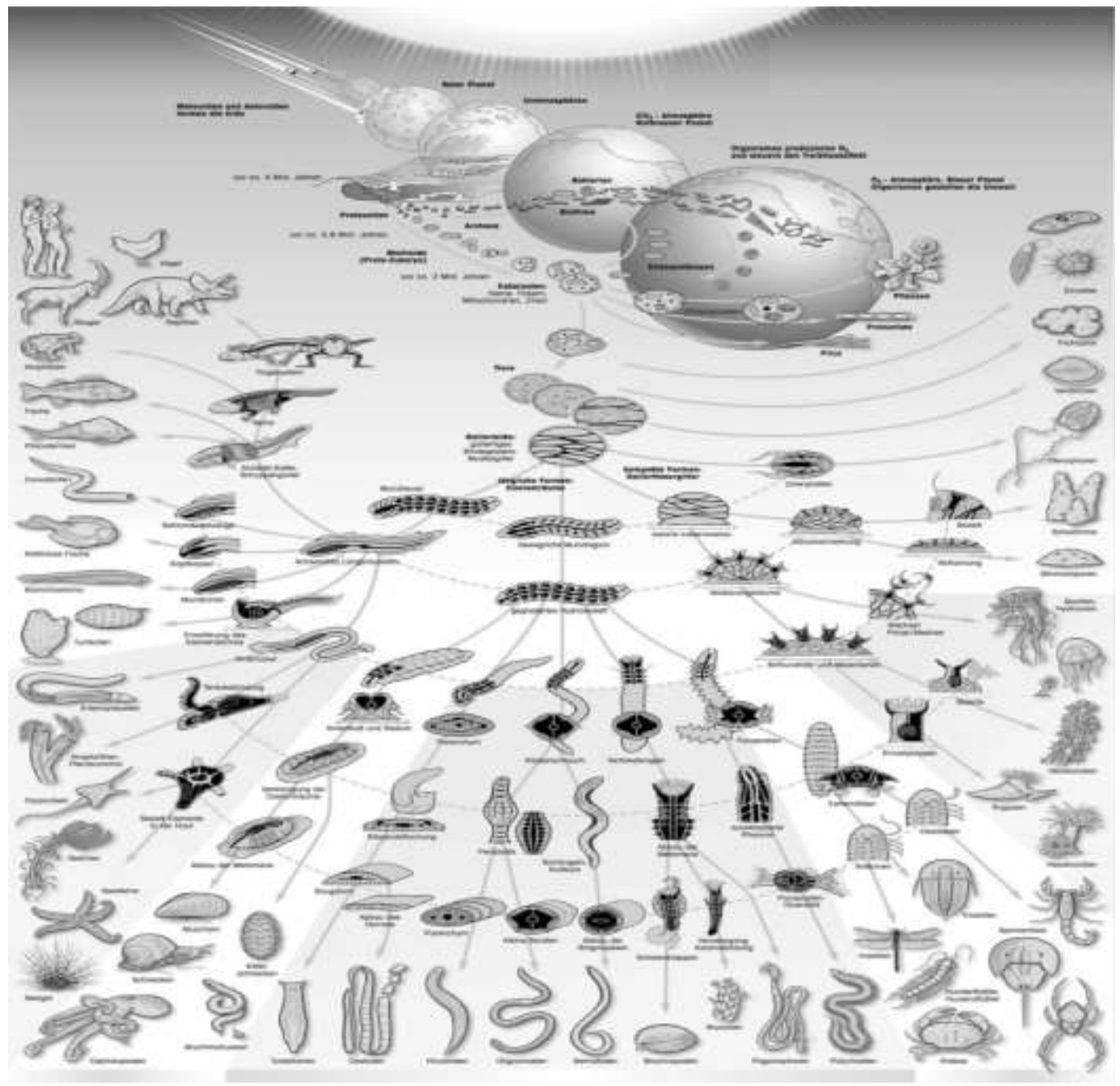

Figure 8: Evolution of animal species in the planet ${ }^{12}$

Stuff and organization being the same all the time, there is only one structural difference that is relevant: complexity. As we have seen above, this is deciding upon the explicit forms that are created in the long run. In the following we see a small selection of forms. 


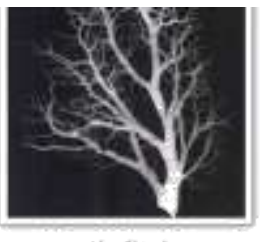

Disodiven

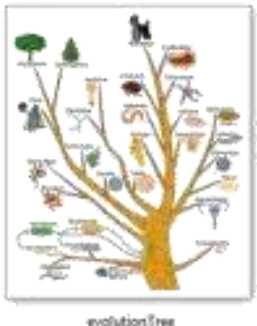

montiontre

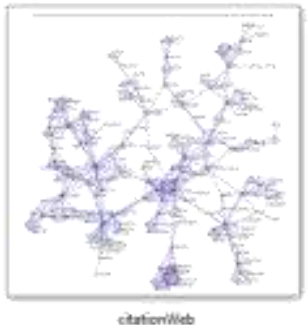

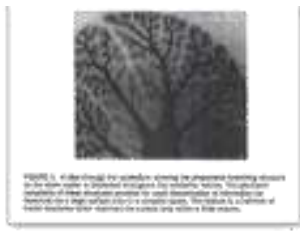

cantevilen
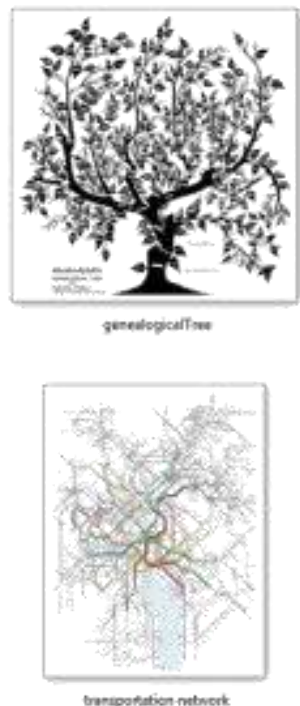
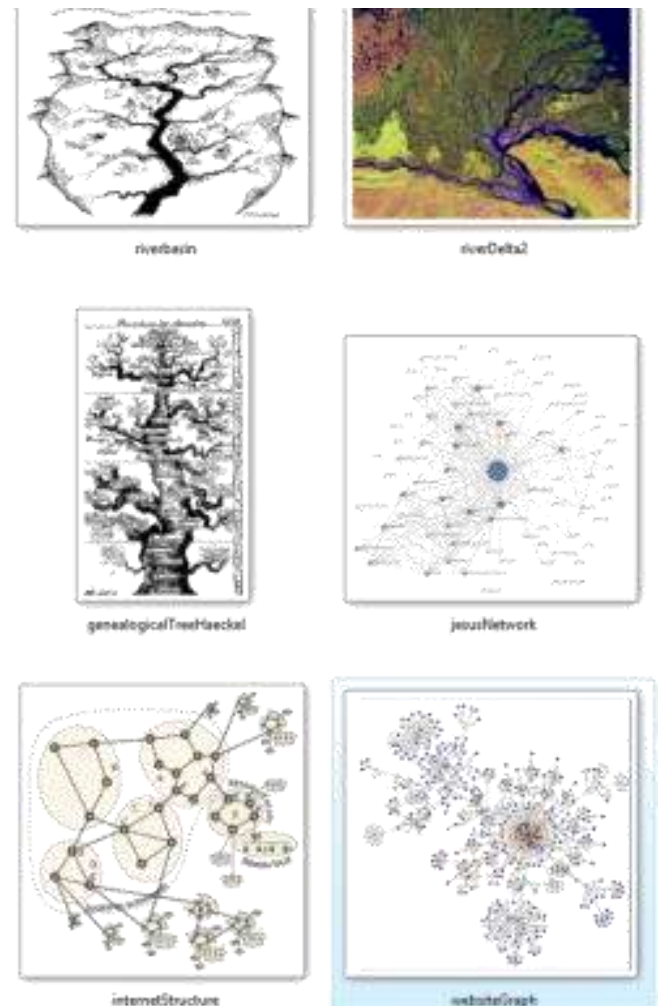

Figure 9: All the tree networks above show empirically observable Pareto-Zipf-Mandelbrot (parabolic fractal) statistical structure. ${ }^{13}$

It should be apparent by now that the modern picture of worldly evolution is not very far from the ancient viewpoint of substance metaphysics. In a moderately modified version of the originally Aristotelian approach, Spinoza visualizes the world as a substance that can be expressed by an infinity of attributes. But human beings have only access to two of these attributes, called matter and mind, respectively, and it is by this characteristic of their mode of being that they are able to model the world according to their (modest) purposes. But obviously, they do not deal with the truth, because the world is not as it is being observed. Hence, this perspective is still ours today. The crucial difference might be the fact that the entities qualifying for us as attributes are energy-mass and information-structure, respectively, rather than matter and mind.

\section{Social Networks}

However, things change considerably when we encounter social networks: This is mainly because it is the human being only that signifies the world and attaches meaning to it by applying various modelling techniques. In other words: The concept of network itself is a human invention. Hence, in nature, there is no network in the strict sense, but things in nature, as far as human beings can cognitively grasp them at all, are modelled such that they are visualized in terms of a variety of networks: Human beings act for instance on the given background of their geography. The latter is determining communication mainly by means of available transport routes that define the infra-structure of the environment. Hence, on this level, life, human or not, is still depending on physical aspects within the biological framework. It can thus be shown that one of the cradles of human civilization, the social space of the ancient Greek culture, is not a coincidental location of emergence: Instead, the geographical contours of this space strongly support the emergence of a network of poleis in the first place, because the organizational structure of an ancient polis can be visualized as a direct consequence (mapping!) of the de-centralized geography of a fractal distribution of islands.

13 Ibid. 


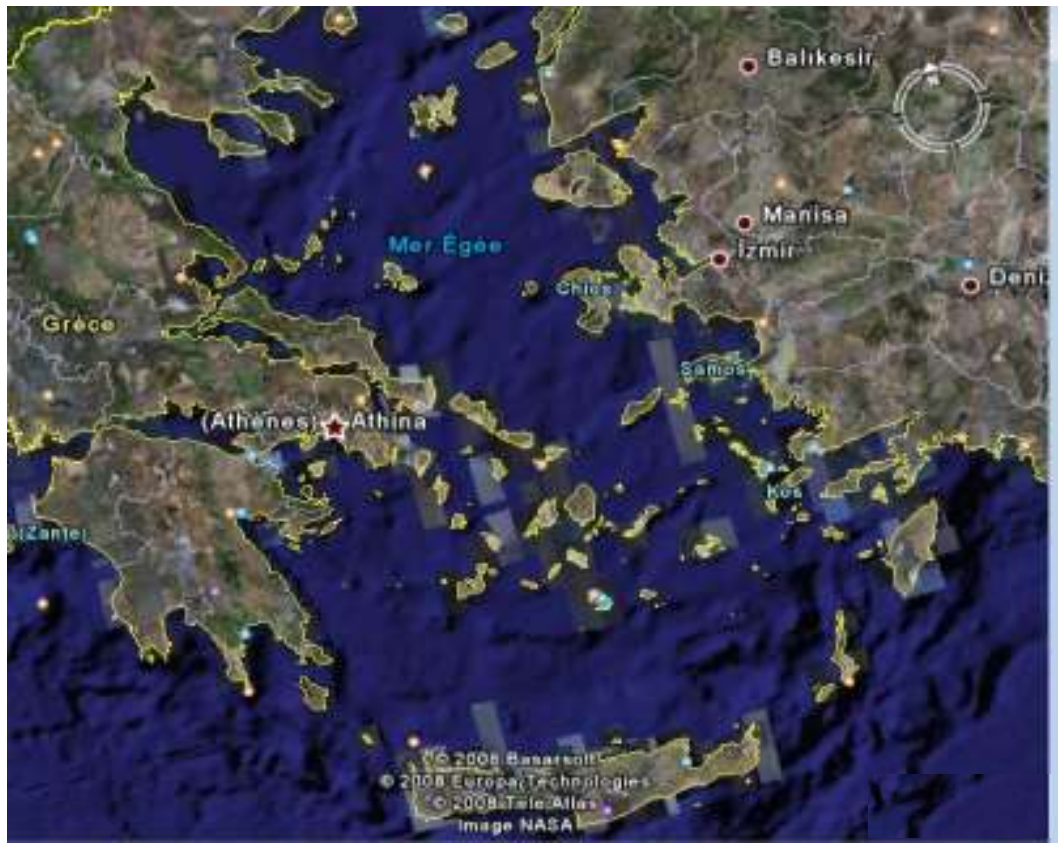

Figure 10: island size distribution are of the PZM Pareto-Zipf-Mandelbrot type (parabolic fractal). ${ }^{14}$

Note that beside the geographical network, human beings also rely on explicitly biological networks whose products they consume. A seaside network of this type is displayed in the following:

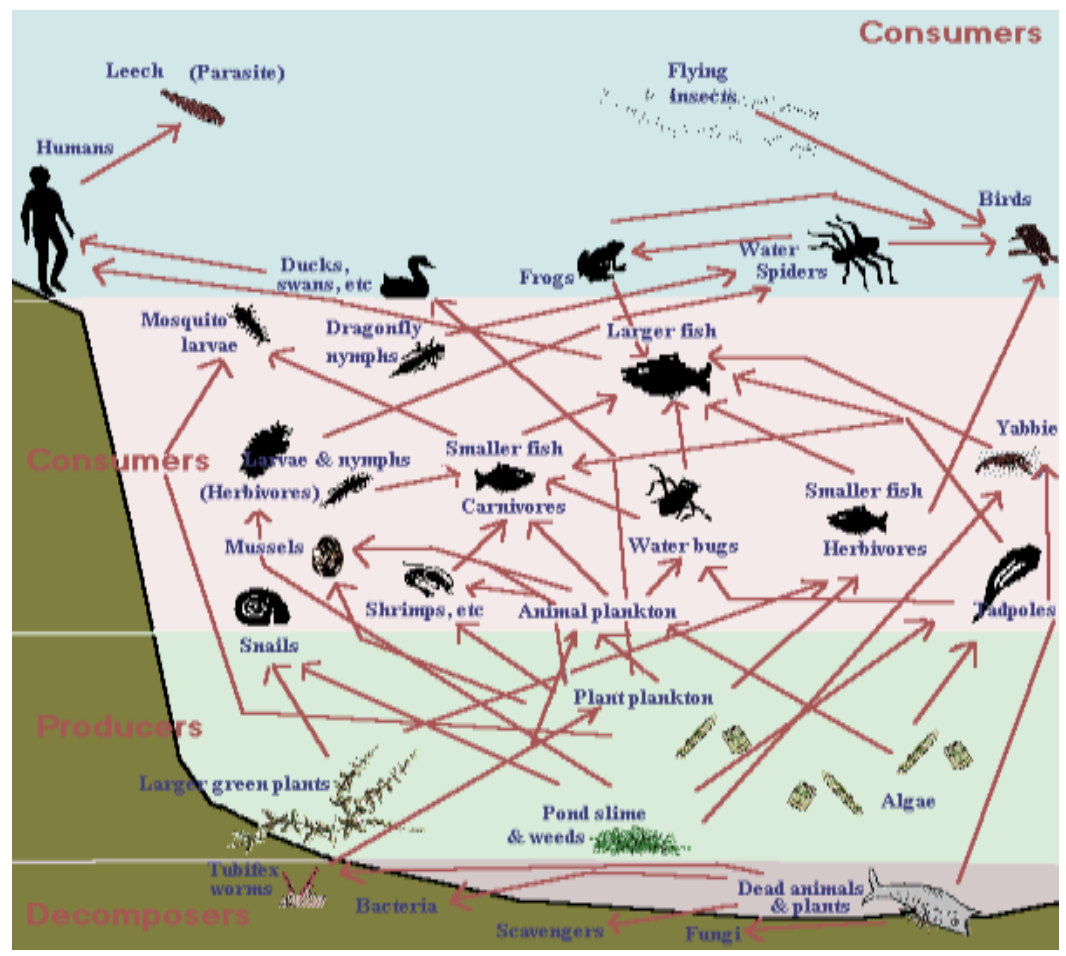

Figure 11: Biomass-size distribution of aquatic ecosystems (trophic web or foodweb). ${ }^{15}$

14 Ibid.

15 Ibid. 
Again, though depending on the modes of consumption organized within the biological submarine network, human beings stand apart somehow: not only, because they are not beings of the sea and do only participate in what other beings have organized themselves - but especially, because they can actually mode/the network in which they are participating. Obviously, the straightforward idea is to apply these models to the community of human beings itself. But the point is that these models are simply mappings (or pictures) and relate to the concrete original as a picture relates to the object it is a picture of. In fact, by applying models, human beings apply nothing but their own cognitive capacity. Hence, from the beginning on, the vital characteristics of human beings are implemented into these models in the first place: This is why observation of animals in the sea entails a large portion of self-observation. Modelling of the seaside eco-system means modelling of an alien world according to human criteria. Hence, the results, if in turn applied back to human conditions, constitute a sort of self-fulfilling prophecy. In fact, the perspective is even more restricted, because while we are talking of human beings, we actually talk of social hubs only, as we can clearly recognize from the next picture.

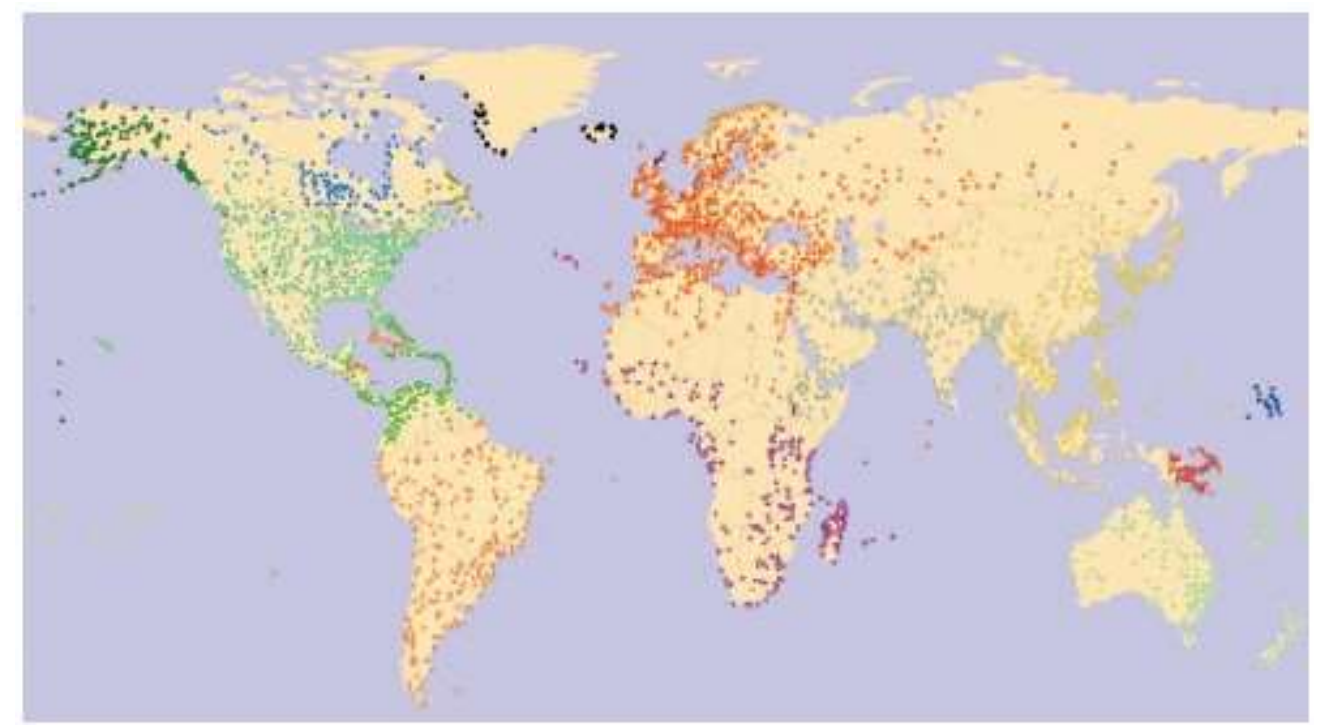

Figure 12: Communities in the giant component of the worldwide air transportation network. Each node represents a location, and each color corresponds to a community. ${ }^{16}$

So this is chiefly the human perspective: What we call observation is the result of cognitive work that is performed according to the boundary conditions of human biological capacities. What we call mode/ is the reconstruction of all these results including the interpolation and extrapolation of details which have been left out in the first place or cannot be actually observed. Models are clustered to form theories, and the set of available theories constitutes the present picture humans have of this world. Within this framework everything is modelled, especially what is not human itself. Nevertheless, the intrinsic self-reference of modelling guarantees that all what is non-human in the world is treated according to what is human. In other words: What we thus observe in a human manner is nothing but a specifically human world, because what we derive from these observations and assemble to the architecture of theories is itself constituted in a characteristically human way. Obviously: What we have in the end, is not the world as it really is, but the world as it is being cognitively perceived according to the human modality. We never deal with reality explicitly, we only deal with modality.

16 Ibid. 


\section{Conclusions}

First of all, there is a rather technical conclusion: We notice that the concepts of space(-time), network, and system form a conceptual triade that characterizes the epistemological aspect of human beings. Networks constitute the dynamical skeleton and/or circulation of a system. If we define a system as a totality of components that interact with each other such that they can be visualized as a conceptual unity, then we realize that the tenor lies on the word "interact". Hence, the sub-totality of interactions in a system is expressed as a network. Interaction and communication can be utilized synonymously for "exchange of information". A network is thus constituting the dynamics that defines the characteristic properties of a system. On the other hand, what we call space is the range of interactions as defined by the network. Hence, space can only be defined reasonably, if there are objects that interact. (Obviously, there is no empty space.) Similarly, time is the sequence in which the system updates itself. Visualized in terms of categories, this means that nodes and links (agents and their operations) are the objects and morphisms, while space and time as range of interactions (domain of dependence) and updating sequence, respectively, define the qualities of the interactions (they define the system's action). Because the operators in a system (objects) can also be visualized as agents, this action is always meaningful and thus intentional. It follows the consequences of the updating results and hence relies on reflexion which is essentially the self-observation of a system with respect to its environment. So in a mild sense of the word, systems can always be visualized as reflexive as well as active. Note that the conceptual triade of epistemological type: space-network-system, corresponds directly to another conceptual triade of ontologica/ type defined by cognition-communication-cooperation. In other words: Because human beings are constituted in a cognitive manner, there is space(-time) - in order to make sure that the human capacity for observations suffices by arranging observed events in a well-defined spatial and temporal order - because human beings have to communicate in order to secure their own orientation within the observable world, there is network - because they must be able to directly interact with the non-human environment (in utilizing energy or stuff) and because they do so by means of cooperation, there is system. It is in fact the type of system that is deciding upon any possible strategic routes to be taken.

This can be seen in the following way: The (actually quite ancient) idea is that form must be arranged in harmony with content. For us here, this means that the explicit management of systems must be an outcome of the results developed before as to the system's structure, comprising characteristic spaces, times, networks of communication, and so forth. Hence, the determination of an adequate form (what the Stoic philosophers called a form which is adequate with respect to nature: kátà physin) depends on the evaluation of cognition. It is thus a primarily aesthetic activity. On the other hand, life is determined by the results of what we call design: Human beings have to behave adequately according to what they know. And in an existential sense, human behaviour is always design in fact. Hence, ethics (which is the lore of adequate behaviour) demands criteria for adequacy that are essentially grounded in aesthetics. This is the old idea of kalokagathía (from: kalós = good, and ágathos = beautiful): namely to produce the desired harmony such as to fit the one to the other.

For the system's perspective this idea has been illustrated somewhat by the Greek philosopher Heraclitus: When asked by his fellow citizens for his opinion on how to achieve harmony in polis life (homonoia = civic harmony), he simply kept silent, took a cup and mixed water and barley flour, adding some mint to make a mixture that follows the recipe for the kykeon (the ritual drink when at the Delphi oracle), drank it and went away, still without speaking. ${ }^{17}$ The message is clear: The mixture remains stable only when permanently stirred. In other words: Harmonic systems (which in science are usually called resilient) based on the state of communication within their (social) networks can be achieved, if they are designed within the boundary conditions of the metastable range of their state space. Hence, there is permanently diverging (and thus conflicting) motion among the components of a system that can only stabilize, if it never stops. This calls for a permanent stirring. The reader is invited to look for practical examples (e.g. as far as the intercultural discourse is concerned). But it should be quite obvious that it is the perspective of networks that grounds praxis on a new insight: Hence, the onto-epistemological viewpoints as to networks carrries explicit ethical connotations, in fact. Moreover, at

17 I am paraphrasing Nicole Loraux who quotes Plutarch's Moralia in: The Divided City. Zone Books, N.Y., 2006, 109. 
the same time, it is actually the main practical field of applications (namely, ICT and Society) within which these connotations unfold in a most innovative as well as concrete manner.

\section{References}

Baez, John: Quantization of Area. The Plot Thickens. http://math.ucr.edu/home/baez/area.pdf. Accessed 2012/07/12.

Barabasi, Albert-László: Linked. The New Science of Networks. Perseus, Cambridge (Mass.), 2002.

Bordalier Institute. www.bordalierinstitute.com. Accessed 2012/07/12

Bornholdt, Stefan, Heinz Georg Schuster (eds.): Handbook of Graphs and Networks, Wiley-VCH, Weinheim, 2003.

Loraux, Nicole: The Divided City. Zone Books, N.Y., 2006.

Zimmermann, Rainer E.: Netzwerkstrukturen und die Emergenz des Neuen. In: Johannes Ehrhardt (ed.), Netzwerk-Dimensionen, Datacom, Bergheim, 1992, 70-85.

Wikipedia: Graph Theory. http://en.wikipedia.org/wiki/Graph theory. Accessed 2012/07/12

Zimmermann, Rainer E.: System des transzendentalen Materialismus, Mentis, Paderborn, 2004. 